\title{
Treatment Outcomes of Patients with Multidrug-Resistant Tuberculosis: Comparison of Pre- and Post-Public-Private Mix Periods
}

\author{
Yewon Kang, M.D. ${ }^{1}{ }^{(\mathbb{D}}$, Eun-Jung Jo, M.D. ${ }^{2}$, Jung Seop Eom, M.D. ${ }^{2,3}$, Mi-Hyun Kim, M.D. ${ }^{2,3}$, Kwangha \\ Lee, M.D. ${ }^{2,3}$, Ki Uk Kim, M.D. ${ }^{2,3}$, Hye-Kyung Park, M.D. ${ }^{2,3}$, Min Ki Lee, M.D. ${ }^{2,3}$ and Jeongha Mok, \\ M.D. ${ }^{2,3,4}$ iD \\ ${ }^{1}$ Department of Internal Medicine, VHS Medical Center, Busan, ${ }^{2}$ Department of Internal Medicine, Pusan National University \\ Hospital, Busan, ${ }^{3}$ Pusan National University School of Medicine, Busan, ${ }^{4}$ Biomedical Research Institute, Pusan National \\ University Hospital, Busan, Republic of Korea
}

Background: This study compared the treatment outcomes of patients with multidrug-resistant tuberculosis (MDRTB) before and after the implementation of public-private mix (PPM). Factors affecting treatment success were also investigated.

Methods: Data from culture-confirmed pulmonary MDR-TB patients who commenced MDR-TB treatment at Pusan National University Hospital between January 2003 and December 2017 were retrospectively reviewed. Patients were divided into two groups in terms of PPM status: pre-PPM period, patients who commenced MDR-TB treatment between 2003 and 2010; and post-PPM period, patients treated between 2011 and 2017.

Results: A total of 176 patients were included (64 and 112 in the pre- and post-PPM periods, respectively). $36.9 \%$ of the patients were resistant to a fluoroquinolone or a second-line injectable drug, or both. The overall treatment success rate was $72.7 \%$. The success rate of post-PPM patients was higher than that of pre-PPM patients $(79.5 \%$ vs. $60.9 \%$, $\mathrm{p}=0.008)$. Also, loss to follow-up was lower in the post-PPM period ( $5.4 \%$ vs. $15.6 \%, \mathrm{p}=0.023$ ). In multivariate regression analysis, age $\geq 65$ years, body mass index $\leq 18.5 \mathrm{~kg} / \mathrm{m}^{2}$, previous TB treatment, bilateral lung involvement, and extensively drug-resistant (XDR)- or pre-XDR-TB were associated with poorer treatment outcomes. However, the use of bedaquiline or delamanid for $\geq 1$ month increased the treatment success.

Conclusion: The treatment success rate in MDR-TB patients was higher in the post-PPM period than in the pre-PPM period, particularly because of the low rate of loss to follow-up. To ensure comprehensive patient-centered PPM in South Korea, investment and other support must be adequate.

Keywords: Multidrug-Resistant Tuberculosis; PPM; Public-Private Mix; South Korea; Treatment Outcome

Address for correspondence: Jeongha Mok, M.D.

Department of Internal Medicine, Pusan National University Hospital, 179 Gudeok-ro, Seo-gu, Busan 49241, Republic of Korea

Phone: 82-51-240-7889, Fax: 82-51-254-3127, E-mail: mokgamokga@gmail.com

Received: Aug. 8, 2020, Revised: Sep. 29, 2020, Accepted: Oct. 28, 2020, Published online: Oct. 28, 2020

@(c) it is identical to the Creative Commons Attribution Non-Commercial License (http://creativecommons.org/licenses/by-nc/4.0/). 


\section{Introduction}

Multidrug-resistant tuberculosis (MDR-TB) constitutes a major global obstacle when seeking to eliminate tuberculosis (TB), and is also a significant public health problem ${ }^{1}$. Treatment is challenging; long-term use of second-line anti-TB drugs that are more toxic and less effective than first-line drugs is essential ${ }^{2}$. Treatment outcomes remain unsatisfactory. The proportion of MDR-TB patients in a 2016 global cohort who successfully completed treatment was only $56 \%^{3}$. The treatment success rate of extensively drug-resistant (XDR)-TB patients is very poor; only $39 \%$ of such patients successfully completed treatment in $2016^{3}$.

Despite national efforts to control TB, South Korea has the highest TB incidence of all member countries of the Organization for Economic Co-operation and Development, with a total TB incidence of $65.9 / 100,000$ in $2018^{4,5}$. In $2018,3.2 \%$ of new cases and $9.2 \%$ of previously treated cases had multidrugresistant (MDR)- or rifampicin-resistant (RR)-TB; 618 patients with MDR-TB were recorded by the Korean National TB Surveillance System ${ }^{3,5}$. The treatment success rates of MDR/RRand XDR-TB in 2016 were only 66 and 58\%, respectively ${ }^{3}$.

Traditionally, public health centers have played major roles in the treatment and management of TB patients; the centers are part of the national TB control program of South Korea. However, when the National Health Insurance system was extended to cover almost the entire South Korean population in 1989, the proportion of TB patients treated by the private sector began to increase steadily (from 54\% in 2001 to $96 \%$ in 2018) ${ }^{5,6}$. However, the treatment outcomes of TB patients treated in the private sector have been poorer than those of patients visiting public health centers. A lack of systematic patient management and inappropriate anti-TB regimens are the principal causes of poor treatment outcomes in the private sector $^{4,7-9}$.

To overcome these problems, South Korea implemented a public-private mix (PPM) as a pilot program based on World Health Organization (WHO) recommendations in 2007, and expanded it nationwide in $2011^{9-11}$. To ensure the comprehensive management of TB patients, trained TB-specialist nurses have been dispatched to private hospitals throughout South Korea. PPM features patient management, including monitoring during the entire length of each treatment, case holding, counseling if adverse drug reactions emerge, health education, contact tracing, and financial support of TB patients ${ }^{4,9}$. In 2017, 127 private hospitals were engaged in PPM in South Korea, and about $70 \%$ of new TB patients were treated in such hospitals ${ }^{9}$.

Positive PPM outcomes have been reported globally ${ }^{4,6,12}$. In South Korea, however, limited data are available on treatment outcomes of MDR-TB patients after the implementation of PPM. This study compared the treatment outcomes of patients with MDR-TB before and after the implementation of PPM. Factors affecting treatment success were also investigated.

\section{Materials and Methods}

\section{Study design and subjects}

This retrospective cohort study was conducted at Pusan National University Hospital (PNUH), a university-affiliated tertiary care hospital in Busan, South Korea; this private hospital has 1,400 beds. PNUH is well-equipped for TB diagnosis and treatment, with TB specialists on staff, an advanced laboratory, and negative-pressure rooms. PNUH serves as a referral hospital for all provincial patients with MDR-TB.

All culture-confirmed pulmonary MDR-TB patients who commenced MDR-TB treatment at PNUH between January 2003 and December 2017 were included. Rifampicin (RIF)monoresistant TB was considered to be MDR-TB. All MDR-TB cases were confirmed using either culture-based, phenotypic drug susceptibility testing (DST) or molecular DST including a line probe assay (LPA) or the Xpert MTB/RIF assay. Patients with extra-pulmonary TB only, or for whom phenotypic DST data were lacking, were excluded. The patients were divided into two groups by PPM status: pre-PPM period, patients who commenced MDR-TB treatment between 2003 and 2010; and post-PPM period, patients treated between 2011 and 2017 . Although PNUH had engaged in PPM since 2009, there was some early confusion in its implementation; thus, 2011 (the year in which PPM expanded nationwide) was chosen as the year of PPM commencement.

The study was reviewed and approved by the Institutional Review Board of PNUH (H-2004-025-090). The need for informed consent was waived given the observational retrospective nature of the study. Our work had no impact on patient diagnosis or treatment.

\section{Data collection}

The following data were collected from medical records: age, sex, height, body weight, comorbidities, smoking status, previous treatment for $\mathrm{TB}$, initial sputum acid-fast bacilli (AFB) smear and laboratory results, DST results, and chest radiograph and computed tomography findings. The diagnostic and treatment modalities were investigated, including bronchoscopy, molecular DST, and treatment regimens (including surgery). Total treatment durations and final outcomes were also evaluated.

\section{Definitions}

MDR-TB was defined as TB resistant to both isoniazid (INH) and RIF; XDR-TB as MDR-TB further resistant to any fluoro- 
quinolone (FQ) and at least one of the three second-line injectable drugs (SLIDs; kanamycin [KM], amikacin [AMK], and capreomycin $[\mathrm{CM}]$ ); and pre-XDR-TB as MDR-TB further resistant to either an FQ or any SLID but not both ${ }^{13}$. The patients were classified into two groups by treatment history: new patients who had never been treated or who had taken anti-TB drugs for $<1$ month, and previously treated patients who had received anti-TB drugs for $\geq 1$ month $^{13}$. Treatment outcomes were categorized in accordance with the WHO definitions as follows: cured, treatment completed, treatment failed, died, lost to follow-up, or not evaluated ${ }^{13}$. Treatment success was defined as the sum of cured and treatment completed. All other treatment outcomes were considered unfavorable.

\section{DST and MDR-TB treatment}

All Mycobacterium tuberculosis isolates were sent to the Korean Institute of Tuberculosis for phenotypic DST. The drug susceptibility of the M. tuberculosis isolates was assessed using the absolute concentration method in Lowenstein-Jensen medium. The drugs included in the phenotypic DST were INH, RIF, ethambutol, rifabutin, streptomycin (SM), AMK, KM, CM, ofloxacin (OFX), levofloxacin (LFX), moxifloxacin (MFX), prothionamide (PTO), cycloserine (CS), and paraaminosalicylic acid. Pyrazinamide (PZA) susceptibility was determined using the pyrazinamidase test. Tests for resistance to AMK and LFX have been available since 2007 and 2009, respectively. The LPA for INH and RIF (GenoType MTBDRplus; Hain Lifescience, Nehren, Germany; or AdvanSure MDR-TB

Table 1. Baseline characteristics of all patients

\begin{tabular}{|c|c|c|c|c|}
\hline Characteristic & $\begin{array}{c}\text { Total } \\
(\mathrm{n}=176)\end{array}$ & $\begin{array}{c}\text { Pre-PPM period } \\
\quad(n=64)\end{array}$ & $\begin{array}{l}\text { Post-PPM period } \\
\quad(n=112)\end{array}$ & p-value* \\
\hline Age, yr & $45.0(31.0-58.0)$ & $41.0(29.3-55.8)$ & $47.5(35.3-62.8)$ & 0.027 \\
\hline Male sex & $108(61.4)$ & $37(57.8)$ & $71(63.4)$ & 0.465 \\
\hline $\mathrm{BMI}, \mathrm{kg} / \mathrm{m}^{2}$ & $21.2(19.3-23.1)$ & $20.8(19.5-22.7)$ & $21.4(19.2-23.3)$ & 0.427 \\
\hline HIV positivity & $1(0.6)$ & $1(1.6)$ & $0(0)$ & 0.364 \\
\hline \multicolumn{5}{|l|}{ Comorbidities } \\
\hline Diabetes mellitus & $41(23.3)$ & $19(29.7)$ & $22(19.6)$ & 0.129 \\
\hline Malignancy & $22(12.5)$ & $9(14.1)$ & $13(11.6)$ & 0.636 \\
\hline Chronic liver disease & $12(6.8)$ & $4(6.3)$ & $8(7.1)$ & $>0.999$ \\
\hline Chronic lung disease & $10(5.7)$ & $2(3.1)$ & $8(7.1)$ & 0.331 \\
\hline Cardiovascular disease & $10(5.7)$ & $1(1.6)$ & $9(8.0)$ & 0.096 \\
\hline Chronic kidney disease & $8(4.5)$ & $2(3.1)$ & $6(5.4)$ & 0.712 \\
\hline Ever-smoker & $81(46.0)$ & $27(42.2)$ & $54(48.2)$ & 0.440 \\
\hline Previous TB treatment & $91(51.7)$ & $44(68.8)$ & $47(42.0)$ & 0.001 \\
\hline Additional extra-pulmonary TB & $10(5.7)$ & $4(6.3)$ & $6(5.4)$ & $>0.999$ \\
\hline Initial, sputum AFB smear positive & $128(72.7)$ & $58(90.6)$ & $70(62.5)$ & $<0.001$ \\
\hline \multicolumn{5}{|l|}{ Radiological findings } \\
\hline Cavities & $114(64.8)$ & $44(68.8)$ & $70(62.5)$ & 0.404 \\
\hline Bilateral lung involvement & $86(48.9)$ & $35(54.7)$ & $51(45.5)$ & 0.243 \\
\hline \multicolumn{5}{|l|}{ Laboratory findings } \\
\hline Serum albumin, $\mathrm{g} / \mathrm{dL}$ & $4.1(3.6-4.4)$ & $3.9(3.5-4.4)$ & $4.2(3.8-4.5)$ & 0.019 \\
\hline Serum total cholesterol, $\mathrm{mg} / \mathrm{dL}^{\dagger}$ & $165.0(140.0-187.0)$ & $166.0(136.3-186.0)$ & $165.0(147.0-192.0)$ & 0.584 \\
\hline \multicolumn{5}{|l|}{ Diagnostic modality } \\
\hline LPA for isoniazid and rifampicin & $68(38.6)$ & $5(7.8)$ & $63(56.3)$ & $<0.001$ \\
\hline Xpert MTB/RIF assay & $59(33.5)$ & $0(0)$ & $59(52.7)$ & $<0.001$ \\
\hline Bronchoscopy & $62(35.2)$ & $29(45.3)$ & $33(29.5)$ & 0.034 \\
\hline
\end{tabular}

Values are presented as median (interquartile range) or number (\%).

${ }^{*}$ Comparison between the pre- and post-PPM periods. ${ }^{\dagger} \mathrm{n}=155$.

PPM: public-private mix; BMI: body mass index; HIV: human immunodeficiency virus; TB: tuberculosis; AFB: acid-fast bacilli; LPA: line probe assay. 
GenoBlot assay; LG Life Sciences, Seoul, Korea) and the Xpert MTB/RIF assay (Cepheid, Sunnyvale, CA, USA) were introduced at PNUH in 2007 and 2012, respectively. All tests were performed according to the manufacturers' instructions.

Treatment regimens were individualized based on the DST results in line with the Korean guidelines. The regimens and treatment durations of these guidelines are similar to those of the WHO guidelines: at least four effective second-line antiTB drugs with or without PZA for at least 20 months ${ }^{14-18}$. Most MDR-TB treatment regimens included an FQ and a SLID if resistance to or intolerance of such drugs was absent. Linezolid (LZD) was introduced at PNUH in 2005 as a component of MDR-TB treatment. Bedaquiline (BDQ) and delamanid
(DLM) were introduced at PNUH in 2015.

\section{PPM activities}

In the time since PPM commencement, all MDR-TB patients began treatment during enforced hospitalization in a negative-pressure room until they became non-infectious (e.g., until the sputum smear became negative); the required duration of forced hospitalization is at least 2 weeks. Anti-TB drugs were administered under directly observed therapy (DOT) during hospitalization but were self-administered after discharge. After discharge, PPM nurses continued to monitor the treatment, offering counseling if adverse drug reactions de-

Table 2. Drug resistances of all patients

\begin{tabular}{|c|c|c|c|c|}
\hline Variable & $\begin{array}{c}\text { Total } \\
(n=176)\end{array}$ & $\begin{array}{l}\text { Pre-PPM period } \\
\quad(n=64)\end{array}$ & $\begin{array}{l}\text { Post-PPM period } \\
\qquad(n=112)\end{array}$ & p-value* \\
\hline \multicolumn{5}{|l|}{ Resistance on phenotypic DST ${ }^{\dagger}$} \\
\hline Isoniazid & $165 / 176(93.8)$ & $62 / 64(96.9)$ & $103 / 112(92.0)$ & 0.332 \\
\hline Rifampicin $^{*}$ & $171 / 176(97.2)$ & $64 / 64(100)$ & $107 / 112(95.5)$ & 0.160 \\
\hline Rifabutin & $128 / 176(72.7)$ & $49 / 64(76.6)$ & $79 / 112(70.5)$ & 0.388 \\
\hline Ethambutol & $112 / 176(63.6)$ & $39 / 64(60.9)$ & $73 / 112(65.2)$ & 0.574 \\
\hline Pyrazinamide ${ }^{\S}$ & $71 / 176(40.3)$ & $30 / 64(46.9)$ & $41 / 112(36.6)$ & 0.182 \\
\hline Ofloxacin & $47 / 176(26.7)$ & $14 / 64(21.9)$ & $33 / 112(29.5)$ & 0.274 \\
\hline Levofloxacin & $31 / 141(22.0)$ & $4 / 29(13.8)$ & $27 / 112(24.1)$ & 0.232 \\
\hline Moxifloxacin & $30 / 176(17.0)$ & $7 / 64(10.9)$ & 23/112 (20.5) & 0.103 \\
\hline Streptomycin & $48 / 176(27.3)$ & $13 / 64(20.3)$ & $35 / 112(31.3)$ & 0.117 \\
\hline Amikacin & $23 / 158(14.6)$ & $8 / 46(17.4)$ & $15 / 112(13.4)$ & 0.517 \\
\hline Kanamycin & $36 / 176(20.5)$ & $18 / 64(28.1)$ & $18 / 112(16.1)$ & 0.057 \\
\hline Capreomycin & $25 / 176(14.2)$ & $10 / 64(15.6)$ & $15 / 112(13.4)$ & 0.683 \\
\hline Prothionamide & $30 / 176(17.0)$ & $10 / 64(15.6)$ & $20 / 112(17.9)$ & 0.705 \\
\hline Cycloserine & $18 / 176(10.2)$ & $8 / 64(12.5)$ & 10/112(8.9) & 0.452 \\
\hline Para-aminosalicylic acid & $55 / 176(31.3)$ & $19 / 64(29.7)$ & $36 / 112(32.1)$ & 0.735 \\
\hline No. of resistant drugs & $5.0(3.0-8.0)$ & $5.0(3.3-8.0)$ & $4.5(3.0-8.0)$ & 0.657 \\
\hline \multicolumn{5}{|l|}{ Resistance level } \\
\hline MDR-TB $^{\| \uparrow}$ & $111(63.1)$ & $38(59.4)$ & $73(65.2)$ & 0.443 \\
\hline Pre-XDR-TB with FQ resistance** & $27(15.3)$ & $7(10.9)$ & $20(17.9)$ & 0.220 \\
\hline Pre-XDR-TB with SLID resistance ${ }^{\dagger \dagger}$ & $18(10.2)$ & $12(18.8)$ & $6(5.4)$ & 0.005 \\
\hline XDR-TB & $20(11.4)$ & $7(10.9)$ & $13(11.6)$ & 0.893 \\
\hline
\end{tabular}

Values are presented as number (\%) or median (interquartile range).

${ }^{*}$ Comparison between the pre- and post-PPM periods. ${ }^{\dagger}$ Number of resistant patients/total tested patients (\%). ${ }^{*}$ Five patients were confirmed to have MDR-TB via molecular DST. ${ }^{\$}$ By the pyrazinamidase test. 'Including 10 patients with rifampicin-monoresistant TB (two in the prePPM period; eight in the post-PPM period). "Multidrug-resistant tuberculosis without additional resistance to fluoroquinolone or a secondline injectable drug. ${ }^{* *}$ Multidrug-resistant tuberculosis resistant to any fluoroquinolone but not to any second-line injectable drug (amikacin, kanamycin, or capreomycin). ${ }^{\dagger}$ Multidrug-resistant tuberculosis resistant to any second-line injectable drug (amikacin, kanamycin, or capreomycin) but not to any fluoroquinolone.

PPM: public-private mix; DST: drug susceptibility test; MDR: multidrug-resistant; TB: tuberculosis; XDR: extensively drug-resistant; FQ: fluoroquinolone; SLID: second-line injectable drug. 
veloped and education via either telephone or face to face. If a patient was non-adherent, the nurse encouraged a hospital visit over the telephone or requested administrative action by a public health center. All hospital stays and visits for diagnosis and treatment were free; the living costs of family members were also supported if the patients were unable to work.

\section{Statistical analysis}

Continuous variables are presented as medians with interquartile ranges, and categorical variables as numbers with percentages. Continuous variables were compared using the Mann-Whitney U test, and categorical variables using the chisquare or Fisher exact test. Significant trends in annual treatment outcomes were examined using the chi-square test for trend. Logistic regression analysis was used to identify factors affecting treatment success. Variables were included in the lo- gistic regression analysis based on the results of the chi-square or Fisher exact test. Factors of interest were also included: sex, diabetes mellitus, presence of cavity, sputum AFB smear positivity, resistance level, and use of LZD, BDQ, or DLM. Variables with a $\mathrm{p}<0.2$ in the univariate analyses were included in the multivariate logistic regression model. Variables were eliminated using the backward stepwise selection method (likelihood ratio). A $p<0.05$ was deemed to reflect statistical significance. All statistical analyses were performed using SPSS Statistics version 22.0 (IBM Corp., Armonk, NY, USA).

\section{Results}

\section{Baseline characteristics}

A total of 185 patients were screened. After application

Table 3. Treatment modalities of all patients

\begin{tabular}{|c|c|c|c|c|}
\hline Variable & $\begin{array}{c}\text { Total } \\
(n=176)\end{array}$ & $\begin{array}{l}\text { Pre-PPM period } \\
\quad(n=64)\end{array}$ & $\begin{array}{c}\text { Post-PPM period } \\
(n=112)\end{array}$ & p-value ${ }^{*}$ \\
\hline \multicolumn{5}{|l|}{ Anti-TB drugs used for $\geq 1 \mathrm{mo}$} \\
\hline Isoniazid & $14(8.0)$ & $9(14.1)$ & $5(4.5)$ & 0.024 \\
\hline Rifabutin & $10(5.7)$ & $5(7.8)$ & $5(4.5)$ & 0.500 \\
\hline Ethambutol & 34 (19.3) & $20(31.3)$ & $14(12.5)$ & 0.002 \\
\hline Pyrazinamide & $116(65.9)$ & 30 (46.9) & $86(76.8)$ & $<0.001$ \\
\hline Ofloxacin & $4(2.3)$ & $4(6.3)$ & $0(0)$ & 0.016 \\
\hline Levofloxacin & $64(36.4)$ & $21(32.8)$ & 43 (38.4) & 0.459 \\
\hline Moxifloxacin & $90(51.1)$ & $42(65.6)$ & 48 (42.9) & 0.004 \\
\hline Any fluoroquinolone ${ }^{\dagger}$ & $152(86.4)$ & $62(96.9)$ & $90(80.4)$ & 0.002 \\
\hline Streptomycin & $52(29.5)$ & $35(54.7)$ & $17(15.2)$ & $<0.001$ \\
\hline Amikacin & $41(23.3)$ & $1(1.6)$ & $40(35.7)$ & $<0.001$ \\
\hline Kanamycin & $56(31.8)$ & $15(23.4)$ & $41(36.6)$ & 0.071 \\
\hline Any injectable drug ${ }^{\ddagger}$ & $143(81.3)$ & $48(75.0)$ & $95(84.8)$ & 0.108 \\
\hline Prothionamide & $141(80.1)$ & $54(84.4)$ & $87(77.7)$ & 0.284 \\
\hline Cycloserine & $149(84.7)$ & $58(90.6)$ & $91(81.3)$ & 0.097 \\
\hline Para-aminosalicylic acid & $71(40.3)$ & $39(60.9)$ & $32(28.6)$ & $<0.001$ \\
\hline Linezolid & $34(19.3)$ & $3(4.7)$ & $31(27.7)$ & $<0.001$ \\
\hline Clofazimine & $9(5.1)$ & $0(0)$ & $9(8.0)$ & 0.027 \\
\hline Bedaquiline & $15(8.5)$ & $0(0)$ & $15(13.4)$ & 0.002 \\
\hline Delamanid & $18(10.2)$ & $0(0)$ & $18(16.1)$ & $<0.001$ \\
\hline No. of drugs used for $\geq 1 \mathrm{mo}$ & $5.0(5.0-6.0)$ & $5.0(5.0-6.0)$ & $5.0(5.0-6.0)$ & 0.255 \\
\hline Surgery to treat TB & $10(5.7)$ & $5(7.8)$ & $5(4.5)$ & 0.500 \\
\hline Total treatment duration, day & $609.0(499.8-706.8)$ & $696.5(486.0-802.8)$ & $608.5(516.5-625.8)$ & 0.001 \\
\hline
\end{tabular}

Values are presented as number (\%) or median (interquartile range).

${ }^{*}$ Comparison between the pre- and post-PPM periods. ${ }^{\dagger}$ Ofloxacin or levofloxacin or moxifloxacin. ${ }^{*}$ Streptomycin or amikacin or kanamycin. PPM: public-private mix; TB: tuberculosis. 
of the above criteria, 176 patients were included in the final analysis (64 patients in the pre-PPM period and 112 in the post-PPM period). Nine patients were excluded: five with extra-pulmonary TB only and four for whom phenotypic DST data were lacking. The baseline characteristics are summarized in Table 1 . The median age was 45 years (interquartile range, $31-58$ years), and 108 patients $(61.4 \%)$ were male. The patients of the post-PPM period were older and had higher serum albumin levels than those of the pre-PPM period. However, the proportions of patients who had received previous TB treatments and those with positive initial sputum AFB smears were higher in the pre-PPM period. More molecular DSTs were performed in the post-PPM period than the prePPM period.

\section{Drug resistance and treatment modality}

Table 2 shows the rates of resistance of all patients to antiTB drugs. Strains from MDR-TB patients exhibited additional resistance to PZA (40.3\%), any FQ (26.7\%), any SLID (21.6\%), PTO (17.0\%), and CS (10.2\%). Pre-XDR- and XDR-TB patients accounted for $36.9 \%$ of all patients. There was no difference in the resistance rates of individual anti-TB drugs or the number of resistant drugs in the pre- and post-PPM periods. However, the proportion of pre-XDR-TB patients exhibiting SLID resistance was lower in the post-PPM period.

The most common anti-TB drugs used in all patients were FQs (OFX, LFX, or MFX; 86.4\%), followed by CS (84.7\%), injectables (SM, AMK, or KM; 81.3\%), PTO (80.1\%), and PZA (65.9\%). Of all patients, $19.3 \%, 10.2 \%$, and $8.5 \%$ were given LZD, DLM, and BDQ, respectively. Ten patients (5.7\%) underwent surgery (Table 3). PZA, AMK, LZD, clofazimine (CFZ), $\mathrm{BDQ}$, and DLM were more commonly prescribed in the postPPM period. The numbers of drugs used for $\geq 1$ month did not differ between the two periods. However, the total treatment duration was shorter in the post-PPM period (Table 3).

\section{Treatment outcomes}

The final treatment outcomes of all patients are listed in Table 4 . The overall treatment success rate was $72.7 \%$. The unfavorable treatment outcomes were as follows: lost to follow-

Table 4. Treatment outcomes of all patients

\begin{tabular}{|c|c|c|c|c|}
\hline Variable & Total & Pre-PPM period & Post-PPM period & p-value* \\
\hline Total & 176 & 64 & 112 & \\
\hline Treatment success & $128(72.7)$ & $39(60.9)$ & $89(79.5)$ & 0.008 \\
\hline Treatment failed & $9(5.1)$ & $5(7.8)$ & $4(3.6)$ & 0.289 \\
\hline Died & $11(6.3)$ & $4(6.3)$ & $7(6.3)$ & $>0.999$ \\
\hline Lost to follow-up & $16(9.1)$ & $10(15.6)$ & $6(5.4)$ & 0.023 \\
\hline Not evaluated & $12(6.8)$ & $6(9.4)$ & $6(5.4)$ & 0.358 \\
\hline${\mathrm{MDR}-\mathrm{TB}^{+*}}^{+1}$ & 111 & 38 & 73 & \\
\hline Treatment success & $85(76.6)$ & $25(65.8)$ & $60(82.2)$ & 0.053 \\
\hline Treatment failed & $5(4.5)$ & $3(7.9)$ & $2(2.7)$ & 0.336 \\
\hline Died & $7(6.3)$ & $2(5.3)$ & $5(6.8)$ & $>0.999$ \\
\hline Lost to follow-up & $9(8.1)$ & $5(13.2)$ & $4(5.5)$ & 0.270 \\
\hline Not evaluated & $5(4.5)$ & $3(7.9)$ & $2(2.7)$ & 0.336 \\
\hline Pre-XDR ${ }^{\S}$ - or XDR-TB & 65 & 26 & 39 & \\
\hline Treatment success & $43(66.2)$ & $14(53.8)$ & $29(74.4)$ & 0.087 \\
\hline Treatment failed & $4(6.2)$ & $2(7.7)$ & $2(5.1)$ & $>0.999$ \\
\hline Died & $4(6.2)$ & $2(7.7)$ & $2(5.1)$ & $>0.999$ \\
\hline Lost to follow-up & $7(10.8)$ & $5(19.2)$ & $2(5.1)$ & 0.106 \\
\hline Not evaluated & $7(10.8)$ & $3(11.5)$ & $4(10.3)$ & $>0.999$ \\
\hline
\end{tabular}

Values are presented as number $(\%)$.

*Comparison between the pre- and post-PPM periods. ${ }^{\dagger}$ Including ten patients with rifampicin-monoresistant TB (two patients in the prePPM period; eight in the post-PPM period). "Multidrug-resistant tuberculosis without additional resistance to fluoroquinolone or a secondline injectable drug. ${ }^{\S}$ Multidrug-resistant tuberculosis resistant to any fluoroquinolone or any second-line injectable drug (amikacin, kanamycin, or capreomycin), but not both.

PPM: public-private mix; MDR: multidrug-resistant; TB: tuberculosis; XDR: extensively drug-resistant. 
up (9.1\%), not evaluated (6.8\%), died (6.3\%), and treatment failed (5.1\%). The treatment success rates of MDR-TB patients lacking additional resistance to FQ and SLID, and those of pre-XDR- and XDR-TB patients, were 76.6 and $66.2 \%$, respectively. The treatment success rate was higher in the post-PPM period $(79.5 \%$ vs. $60.9 \%)$. Of the unfavorable outcomes, the loss to follow-up rate was significantly lower in the post-PPM period (5.4\% vs. $15.6 \%)$. Figure 1 shows the annual trends in the treatment outcomes of all patients. Treatment success increased every year, and the proportions of patients who failed treatment, were lost to follow-up, and who were not evaluated decreased every year.

\section{Factors affecting treatment success}

Comparison of patients whose treatments were successful and those that were not revealed that, in the latter group the body mass index (BMI) and serum albumin level were lower, and the proportions who had undergone prior TB treatment and who exhibited bilateral lung involvement were higher (Table 5). In multivariate regression analysis, age $\geq 65$ years, BMI $\leq 18.5 \mathrm{~kg} / \mathrm{m}^{2}$, previous TB treatment, bilateral lung involvement, and pre-XDR- or XDR-TB were associated with poorer treatment outcomes. However, use of BDQ or DLM for $\geq 1$ month increased the treatment success rate (Table 6).

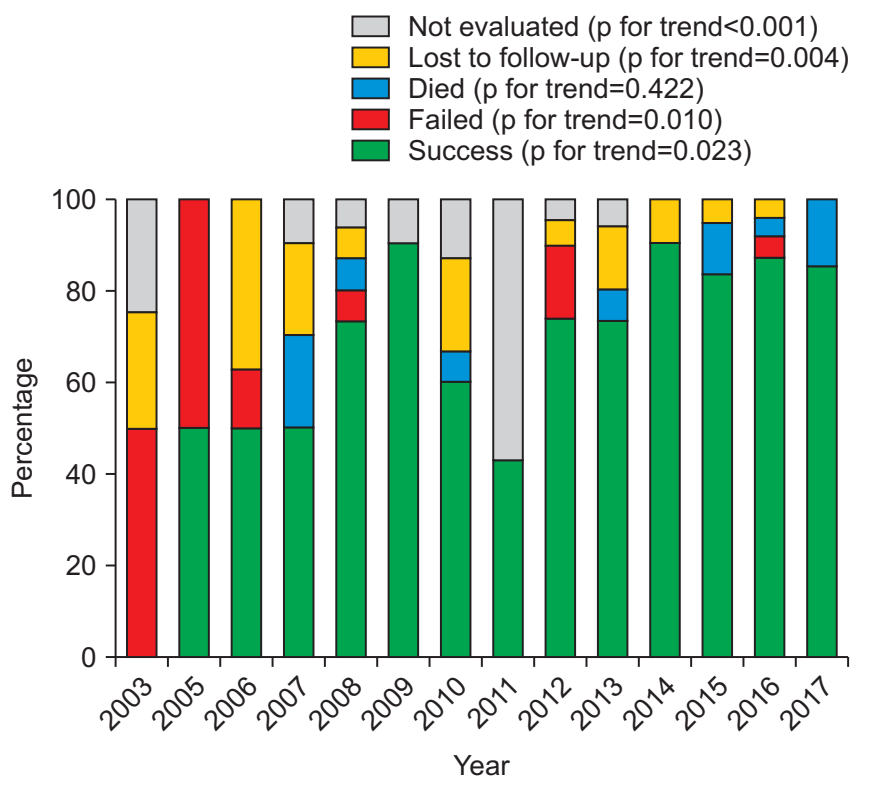

Figure 1. Annual trends in the treatment outcomes of all patients. Data are the annual proportions of all treatment outcomes. No patient commenced on multidrug-resistant tuberculosis treatment in 2004.

\section{Discussion}

It is well-known that loss to follow-up reduces treatment success in MDR-TB patients in South Korea. A recent study employing national registry data showed that although the trend was decreasing, about $10 \%$ of MDR-TB patients are still lost to follow-up annually ${ }^{19}$. In this study, $14 \%$ of MDR-TB patients were transferred out during treatment. However, many of these patients were probably lost to follow-up. This is a serious problem in South Korea, but is not unique to our country. In one global cohort, $15 \%$ of MDR/RR-TB patients were lost to follow-up in $2016^{3}$, risking drug resistance, complications, death, and the community spread of difficult-to-treat pathogens $^{20,21}$. Of the 16 patients lost to follow-up in our study, 11 were positive for M. tuberculosis culture at the time of loss. About half of all MDR-TB patients had no history of prior TB treatment. The proportions of new patients among all MDRTB patients did not decrease annually in an earlier South Korean study ${ }^{22}$. This may be partly explained by the fact that such patients are lost while infectious, and thus spread the disease in the community.

In our study, the treatment success rate of MDR-TB patients was higher in the post-PPM period than in the pre-PPM period, particularly because of the low rate of loss to followup. In terms of patient management, PPM contributed to the decrease in loss to follow-up. However, other factors may be important in the post-PPM period. Many improvements to the national TB control program have been made, and patient socioeconomic status in the post-PPM period has also changed. New or repurposed anti-TB drugs such as LZD, CFZ, BDQ, and DLM were more commonly prescribed in the post-PPM period. These drugs are efficacious, as revealed by a meta-analysis and real-world data ${ }^{23,24}$. Two new drugs, BDQ and DLM, are particularly patient-friendly (few adverse drug reactions); their use was associated with higher treatment success in our study. In the post-PPM period, the proportion of patients with positive sputum AFB smears was lower and the proportion that underwent molecular DST higher. Thus, MDR-TB may have been diagnosed when the bacterial burden was low. Early diagnosis and rapid appropriate treatment may have improved outcomes. Unfortunately, it was impossible to quantify the contribution of PPM to the positive outcome using our pre-post period study design. Further studies with appropriate designs are needed to clarify the impact of PPM on the treatment outcome of MDR-TB patients in South Korea.

Although PPM was successfully implemented in South Korea, several problems remain. First, a full course of DOT is required, as it is crucial for improving treatment adherence and has been recommended by the WHO for all MDR-TB patients ${ }^{17,25}$. In South Korea, however, most MDR-TB patients undergo DOT only during hospitalization. Only selected patients (those receiving new or repurposed drugs, or who do 
Table 5. Comparisons of patients for whom treatment was successful and not

\begin{tabular}{|c|c|c|c|c|}
\hline Variable & $\begin{array}{c}\text { Total } \\
(n=176)\end{array}$ & $\begin{array}{l}\text { Treatment success } \\
\qquad(n=128)\end{array}$ & $\begin{array}{l}\text { Unfavorable outcome } \\
\qquad(n=48)\end{array}$ & p-value* \\
\hline Age, yr & $45.0(31.0-58.0)$ & $43.0(31.0-55.8)$ & $51.5(30.3-68.8)$ & 0.105 \\
\hline Male sex & $108(61.4)$ & $75(58.6)$ & $33(68.8)$ & 0.218 \\
\hline BMI, $\mathrm{kg} / \mathrm{m}^{2}$ & $21.2(19.3-23.1)$ & $21.5(19.5-23.3)$ & $20.3(17.9-22.3)$ & 0.017 \\
\hline \multicolumn{5}{|l|}{ Comorbidities } \\
\hline Diabetes mellitus & $41(23.3)$ & $26(20.3)$ & $15(31.3)$ & 0.126 \\
\hline Malignancy & $22(12.5)$ & $14(10.9)$ & $8(16.7)$ & 0.306 \\
\hline Previous TB treatment & $91(51.7)$ & $57(44.5)$ & $34(70.8)$ & 0.002 \\
\hline Initial, sputum AFB smear positive & $128(72.7)$ & $89(69.5)$ & $39(81.3)$ & 0.120 \\
\hline \multicolumn{5}{|l|}{ Radiological findings } \\
\hline Cavities & $114(64.8)$ & $80(62.5)$ & $34(70.8)$ & 0.303 \\
\hline Bilateral lung involvement & $86(48.9)$ & $52(40.6)$ & $34(70.8)$ & $<0.001$ \\
\hline \multicolumn{5}{|l|}{ Laboratory findings } \\
\hline Serum albumin, g/dL & $4.1(3.6-4.4)$ & $4.2(3.8-4.4)$ & $3.9(3.4-4.4)$ & 0.026 \\
\hline Serum total cholesterol, $\mathrm{mg} / \mathrm{dL}^{\dagger}$ & $165.0(140.0-187.0)$ & $167.0(147.8-186.3)$ & $164.0(133.5-189.5)$ & 0.372 \\
\hline No. of resistant drugs & $5.0(3.0-8.0)$ & $4.0(3.0-8.0)$ & $5.0(3.0-8.8)$ & 0.197 \\
\hline Pre-XDR ${ }^{\ddagger}$ - or XDR-TB & $65(36.9)$ & $43(33.6)$ & $22(45.8)$ & 0.134 \\
\hline Total treatment duration, day & $609.0(499.8-706.8)$ & $618.5(607.0-723.0)$ & $296.0(134.5-544.3)$ & $<0.001$ \\
\hline \multicolumn{5}{|l|}{ Anti-TB drugs used for $\geq 1$ mo } \\
\hline Levofloxacin or moxifloxacin & $151(85.8)$ & $109(85.2)$ & $42(87.5)$ & 0.692 \\
\hline Any injectable drug ${ }^{\S}$ & $143(81.3)$ & $106(82.8)$ & $37(77.1)$ & 0.386 \\
\hline Linezolid & $34(19.3)$ & $28(21.9)$ & $6(12.5)$ & 0.161 \\
\hline Bedaquiline or delamanid & $30(17.0)$ & $25(19.5)$ & $5(10.4)$ & 0.152 \\
\hline No. of drugs used for $\geq 1 \mathrm{mo}$ & $5.0(5.0-6.0)$ & $5.0(5.0-6.0)$ & $5.0(5.0-6.0)$ & 0.541 \\
\hline Surgery to treat TB & $10(5.7)$ & $8(6.3)$ & $2(4.2)$ & 0.730 \\
\hline
\end{tabular}

Values are presented as median (interquartile range) or number (\%).

${ }^{*}$ Comparison between patients for whom treatment was successful and not successful. ${ }^{\dagger} \mathrm{n}=155$. ${ }^{*}$ Multidrug-resistant tuberculosis resistant to any fluoroquinolone or any second-line injectable drug (amikacin, kanamycin, or capreomycin), but not both. ${ }^{\circledR}$ Streptomycin or amikacin or kanamycin.

BMI: body mass index; AFB: acid-fast bacilli; XDR: extensively drug-resistant; TB: tuberculosis.

not adhere to treatment) undergo a full course of DOT after discharge. Second, PPM should be expanded. The loss to follow-up of MDR-TB patients was significantly lower than the national rate in both our hospital and another South Korean PPM hospital ${ }^{19,26}$. MDR-TB patients could be referred to PPM hospitals. Third, PPM requires a more comprehensive approach. Health education, socioeconomic/financial support, emotional/psychosocial assistance (e.g., addressing stigma and discrimination), palliative care, and management of adverse drug reactions would improve treatment adherence ${ }^{27}$. These are essential components of patient-centered care, but are neglected by many national TB programs.

PPM alone is not enough to achieve better treatment outcomes in MDR-TB patients. Shorter, patient-friendly MDR-TB treatment regimens are urgently required. A long treatment duration and adverse drug reactions are well-known risk factors for loss to follow-up ${ }^{1,28,29}$. In addition, an expert MDR-TB committee is required. In practice, a significant proportion of MDR-TB patients respond poorly because of adverse drug reactions or high-level drug resistance. The committee could review treatments and advise attending physicians on appropriate modifications. In Taiwan, the treatment success rate increased and the loss to follow-up rate decreased dramatically after an expert committee was formed ${ }^{30}$.

Our work had several limitations. First, the study was conducted in a single institution with a small number of patients; the results may not be generalizable to South Korea as a whole. Second, a retrospective observational approach can- 
Table 6. Predictors of treatment success

\begin{tabular}{|c|c|c|c|c|c|c|}
\hline \multirow{2}{*}{ Variable } & \multicolumn{3}{|c|}{ Univariate analysis } & \multicolumn{3}{|c|}{ Multivariate analysis } \\
\hline & OR & $95 \% \mathrm{CI}$ & p-value & OR & $95 \% \mathrm{CI}$ & p-value \\
\hline Age $\geq 65 \mathrm{yr}$ & 0.322 & $0.142-0.734$ & 0.007 & 0.189 & $0.070-0.513$ & 0.001 \\
\hline Male sex & 0.643 & $0.318-1.301$ & 0.220 & - & - & - \\
\hline $\mathrm{BMI} \leq 18.5 \mathrm{~kg} / \mathrm{m}^{2}$ & 0.407 & $0.188-0.884$ & 0.023 & 0.312 & $0.127-0.766$ & 0.011 \\
\hline Diabetes mellitus & 0.561 & $0.266-1.184$ & 0.129 & 0.657 & $0.270-1.595$ & 0.353 \\
\hline Previous TB treatment & 0.331 & $0.162-0.675$ & 0.002 & 0.388 & $0.172-0.876$ & 0.023 \\
\hline Cavities & 0.686 & $0.335-1.407$ & 0.304 & - & - & - \\
\hline Bilateral lung involvement & 0.282 & $0.138-0.576$ & 0.001 & 0.328 & $0.148-0.728$ & 0.006 \\
\hline Initial, sputum AFB smear positive & 0.527 & $0.233-1.192$ & 0.124 & 1.256 & $0.455-3.468$ & 0.660 \\
\hline Serum albumin $\leq 3.4 \mathrm{~g} / \mathrm{dL}$ & 0.310 & $0.128-0.751$ & 0.009 & 0.722 & $0.237-2.200$ & 0.567 \\
\hline Pre-XDR* ${ }^{*}$ or XDR-TB & 0.598 & $0.304-1.175$ & 0.136 & 0.340 & $0.147-0.783$ & 0.011 \\
\hline Linezolid use $\geq 1$ mo & 1.960 & $0.756-5.081$ & 0.166 & 2.921 & $0.662-12.886$ & 0.157 \\
\hline Bedaquiline or delamanid use $\geq 1$ mo & 2.087 & $0.750-5.812$ & 0.159 & 5.939 & $1.680-20.991$ & 0.006 \\
\hline
\end{tabular}

*Multidrug-resistant tuberculosis resistant to any fluoroquinolone or any second-line injectable drug (amikacin, kanamycin, or capreomycin), but not both.

OR: odds ratio; CI: confidence interval; BMI: body mass index; TB: tuberculosis; AFB: acid-fast bacilli; XDR: extensively drug-resistant.

not reveal why loss to follow-up occurred; identification of the causes of such loss would improve PPM. Third, we could not track the outcomes of "not evaluated" patients; they may have been lost to follow-up, failed treatment, or died.

In conclusion, the treatment success rate of MDR-TB patients was higher in the post- than pre-PPM period, particularly due to the low rate of loss to follow-up. To ensure comprehensive patient-centered PPM nationwide, adequate financial and human investments are required.

\section{Authors' Contributions}

Conceptualization: Kang Y, Mok J. Methodology: Kang Y, Mok J. Formal analysis: Kang Y, Lee K, Kim KU, Park HK, Lee MK, Mok J. Data curation: Kang Y, Jo EJ, Eom JS, Kim MH, Mok J. Writing - original draft preparation: Kang Y, Mok J. Writing review and editing: Kang Y, Jo EJ, Eom JS, Kim MH, Lee K, Kim KU, Park HK, Lee MK, Mok J. Approval of final manuscript: all authors.

\section{Conflicts of Interest}

No potential conflict of interest relevant to this article was reported.

\section{Funding}

This work was supported by clinical research grant from Pusan National University Hospital in 2020.

\section{References}

1. Orenstein EW, Basu S, Shah NS, Andrews JR, Friedland GH, Moll AP, et al. Treatment outcomes among patients with multidrug-resistant tuberculosis: systematic review and metaanalysis. Lancet Infect Dis 2009;9:153-61.

2. Bastos ML, Lan Z, Menzies D. An updated systematic review and meta-analysis for treatment of multidrug-resistant tuberculosis. Eur Respir J 2017;49:160803.

3. World Health Organization. Global tuberculosis report 2019. WHO/CDS/TB/2019.15. Geneva: World Health Organization; 2019.

4. Kim JH, Yim JJ. Achievements in and challenges of tuberculosis control in South Korea. Emerg Infect Dis 2015;21:1913-20.

5. Korea Centers for Disease Control and Prevention. Annual report on the notified tuberculosis patients in Korea 2018. Cheongju: Korea Centers for Disease Control and Prevention; 2019.

6. Kim HJ, Bai GH, Kang MK, Kim SJ, Lee JK, Cho SI, et al. A public-private collaboration model for treatment intervention to improve outcomes in patients with tuberculosis in the private sector. Tuberc Respir Dis 2009;66:349-57.

7. Hong YP, Kim SJ, Lew WJ, Lee SH, Lee EK. Cohort analyses of the treatment of smear-positive pulmonary tuberculosis pa- 
tients under programme conditions in Korea, 1983-1994. Int J Tuberc Lung Dis 1998;2:365-71.

8. Hong YP, Kim SJ, Lee EG, Lew WJ, Bai JY. Treatment of bacillary pulmonary tuberculosis at the chest clinics in the private sector in Korea, 1993. Int J Tuberc Lung Dis 1999;3:695-702.

9. Go U, Park M, Kim UN, Lee S, Han S, Lee J, et al. Tuberculosis prevention and care in Korea: evolution of policy and practice. J Clin Tuberc Other Mycobact Dis 2018;11:28-36.

10. Cho KS. Tuberculosis control in the Republic of Korea. Epidemiol Health 2018;40:e2018036.

11. World Health Organization. Public-private mix for DOTS: towards scaling up. Geneva: World Health Organization; 2005.

12. Lei X, Liu Q, Escobar E, Philogene J, Zhu H, Wang Y, et al. Public-private mix for tuberculosis care and control: a systematic review. Int J Infect Dis 2015;34:20-32.

13. World Health Organization. Definitions and reporting framework for tuberculosis-2013 revision. WHO/HTM/TB/2013.2. Geneva: World Health Organization; 2013.

14. World Health Organization. Guidelines for the programmatic management of drug-resistant tuberculosis. Geneva: World Health Organization; 2006.

15. World Health Organization. Guidelines for the programmatic management of drug-resistant tuberculosis: emergency update 2008. WHO/HTM/TB/2008.402. Geneva: World Health Organization; 2008.

16. World Health Organization. Guidelines for the programmatic management of drug-resistant tuberculosis: 2011 update. WHO/HTM/TB/2011.6. Geneva: World Health Organization; 2011.

17. World Health Organization. Companion handbook to the WHO guidelines for the programmatic management of drugresistant tuberculosis. WHO/HTM/TB/2014.11. Geneva: World Health Organization; 2014.

18. World Health Organization. WHO treatment guidelines for drug-resistant tuberculosis: 2016 update. WHO/HTM/ TB/2016.04. Geneva: World Health Organization; 2016.

19. Lee M, Han J, Kim YR, Kwak N, Kim JH, Park O, et al. Multidrug-resistant tuberculosis in South Korea: a retrospective analysis of national registry data in 2011-2015. Int J Tuberc Lung Dis 2019;23:850-7.

20. Gandhi NR, Nunn P, Dheda K, Schaaf HS, Zignol M, van Soolingen D, et al. Multidrug-resistant and extensively drugresistant tuberculosis: a threat to global control of tuberculo- sis. Lancet 2010;375:1830-43.

21. Kigozi G, Heunis C, Chikobvu P, Botha S, van Rensburg D. Factors influencing treatment default among tuberculosis patients in a high burden province of South Africa. Int J Infect Dis 2017;54:95-102.

22. Mok JH, Kang BH, Lee T, Lee HK, Jang HJ, Cho YJ, et al. Additional drug resistance patterns among multidrug-resistant tuberculosis patients in Korea: implications for regimen design. J Korean Med Sci 2017;32:636-41.

23. Collaborative Group for the Meta-Analysis of Individual Patient Data in MDR-TB treatment-2017; Ahmad N, Ahuja SD, Akkerman OW, Alffenaar JW, Anderson LF, et al. Treatment correlates of successful outcomes in pulmonary multidrugresistant tuberculosis: an individual patient data meta-analysis. Lancet 2018;392:821-34.

24. Mok J, Kang H, Koh WJ, Jhun BW, Yim JJ, Kwak N, et al. Final treatment outcomes of delamanid-containing regimens in patients with MDR-/XDR-TB in South Korea. Eur Respir J 2019;54:1900811.

25. World Health Organization. Guidelines for establishing DOTS-PLUS pilot projects for the management of multidrugresistant tuberculosis (MDR-TB). WHO/CDS/TB/2000.279. Geneva: World Health Organization; 2000.

26. Kwak N, Kim HR, Yoo CG, Kim YW, Han SK, Yim JJ. Multidrug-resistant tuberculosis over 20 years at a referral hospital in South Korea: trends and outcomes. Int J Tuberc Lung Dis 2019;23:174-80.

27. World Health Organization. Framework for the engagement of all health care providers in the management of drug resistant tuberculosis. WHO/HTM/TB/2015.04. Geneva: World Health Organization; 2015.

28. Johnston JC, Shahidi NC, Sadatsafavi M, Fitzgerald JM. Treatment outcomes of multidrug-resistant tuberculosis: a systematic review and meta-analysis. PLoS One 2009;4:e6914.

29. Holtz TH, Lancaster J, Laserson KF, Wells CD, Thorpe L, Weyer K. Risk factors associated with default from multidrugresistant tuberculosis treatment, South Africa, 1999-2001. Int J Tuberc Lung Dis 2006;10:649-55.

30. Yu MC, Chiang CY, Lee JJ, Chien ST, Lin CJ, Lee SW, et al. Treatment outcomes of multidrug-resistant tuberculosis in Taiwan: tackling loss to follow-up. Clin Infect Dis 2018;67:20210. 